\title{
Endoprothetik beim Rheumatiker
}

Liebe Leserinnen und Leser der arthritis + rheuma,

In der operativen Orthopädie ist die Entwicklung der Endoprothetik an der Hüfte und am Kniegelenk, trotz zahlreicher Rückschläge, eine beispiellose Erfolgsgeschichte. Die überwiegende Mehrzahl der Patienten gewinnt durch die Schmerzbefreiung oder zumindest -reduktion und die Verbesserung der Beweglichkeit und Mobilität erheblich an Lebensqualität. Die Standzeiten dieser Prothesen sind heutzutage sehr gut, sodass der Verschleiß der Gleitpaarung zunehmend an Bedeutung gewinnt.

An der Schulter und am Ellenbogen sind die Komplikationen, trotz der geringeren Belastung der Gelenke der oberen Extremitäten, häufiger. Aber auch hier konnte mit der Einführung der inversen Prothesen an der Schulter und der gekoppelten Ellenbogenprothesen mit erhaltenem „Gelenkspiel“, Stichwort „semiconstrained“, bei guter Primärstabilität der Implantate eine verbesserte Funktion bei geringerer Komplikationsrate erreicht werden.

Am oberen Sprunggelenk kommt es häufig nach anfänglich guter Beweglichkeit durch intra- und periartikuläre Narben und Verkalkungen zum Bewegungsverlust. Der Vorteil der Prothese gegenüber dem Goldstandard der Arthrodese, über eine Dorsalextension das Gangbild zu harmonisieren, geht dadurch verloren oder wird primär, bei falscher Indikationsstellung, gar nicht erst erreicht.

Die Endoprothesen an Hand-, Daumensattel-, Finger- und Zehengelenken werden, aufgrund hoher Komplikationsraten und in der Regel fehlender Überlegenheit in der Vorteil-Risiko-Abwägung gegenüber den konkurrierenden Arthrodesen und Resektionsarthroplastiken, nur bei spezieller Indikation angewendet, sind aber nicht der Goldstandard in der Versorgung destruierter Gelenke.

Die Erkrankungen des rheumatischen Formenkreises führen, auch im Zeitalter der effektiveren Immunsuppression mit Biologika, zur im Vergleich zur Normalbevölkerung vorzeitigen Gelenkdestruktion, auch wenn diese unter wirksamer Therapie heute langsa- mer eintritt als früher. Das Komplikationsrisiko bei der endoprothetischen Versorgung ist beim immunsupprimierten Rheumatiker erhöht. Daneben bestehen spezifische Risiken, wie der serositische Rheumaschub und die Instabilität der oberen HWS mit potenziell letalem Ausgang. Weitere Risiken sind die Osteoporose und Osteomalazie, wodurch periprothetische Frakturen intraoperativ gesetzt werden können, die dann die postoperative Mobilisation verhindern oder zumindest erschweren. Durch die zunehmend auch von den Kostenträgern geforderte Spezialisierung in der orthopädischen Chirurgie, welche in den Endoprothesenzentren ihren Ausdruck findet, liegt der Fokus der endoprothetischen Versorgung auf einer standardisierten Operationstechnik unter biomechanischen Gesichtspunkten. Allgemeinerkrankungen mit spezifischen Risiken werden von diesen Spezialisten, die vorwiegend Arthrosen behandeln, häufig nicht beachtet, negiert oder unterschätzt. Darüber hinaus fehlt in diesem System die ganzheitliche Betrachtung, welche zur Erstellung eines Therapieplanes für den Rheumatiker unerlässlich ist. Dies führt zu planungsbedingten Komplikationen und darüber vermehrt zu Revisionseingriffen, welche in der Hand des orthopädischen Rheumatologen, der als Generalist tätig ist, hätten vermieden werden können.

Sie finden in diesem Themenheft eine Sammlung von Artikeln zur Endoprothetik an Hüfte, Knie, Schulter und Ellenbogen beim Rheumatiker. Ein weiterer Artikel beschreibt die Besonderheiten der Schulterendoprothetik des Adoleszenten mit juveniler Polyarthritis.

Wir wünschen Ihnen bei der Lektüre viel Vergnügen und zahlreiche Erkenntnisse für Ihre tägliche Arbeit in Praxis und Klinik.

Mit freundlichen Grüßen

Ihre

Prof. Dr. Ralph Gaulke

Hannover

Herausgeber

\section{Dr. med. Roger Scholz}

Oschatz

Gastherausgeber

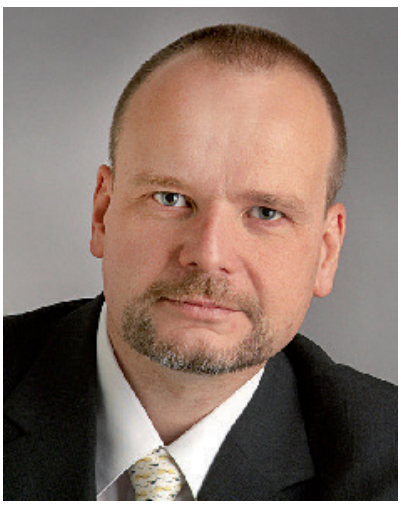

Prof. Dr. Ralph Gaulke

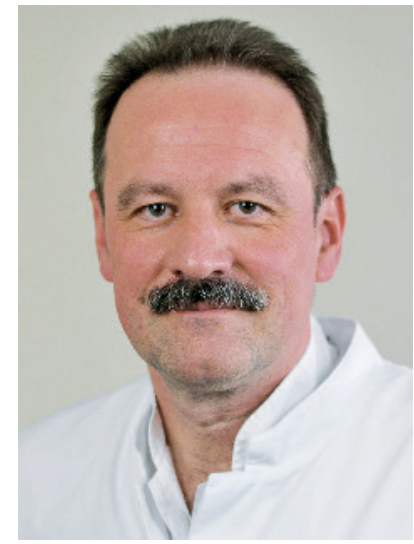

Dr. Roger Scholz 